\title{
The Symplectic Camel and Quantum Universal Invariants: the Angel of Geometry versus the Demon of Algebra
}

\author{
Maurice A. de Gosson* \\ University of Vienna, NuHAG \\ Nordbergstr. 15, 1090 Vienna
}

April 15, 2013

\begin{abstract}
A positive definite symmetric matrix $\sigma$ qualifies as a quantum mechanical covariance matrix if and only if $\sigma+\frac{1}{2} i \hbar \Omega \geq 0$ where $\Omega$ is the standard symplectic matrix. This well-known condition is a strong version of the uncertainty principle, which can be reinterpreted in terms of the topological notion of symplectic capacity, closely related to Gromov's non-squeezing theorem. We show that a recent refinement of the latter leads to a new class of geometric invariants. These are the volumes of the orthogonal projections of the covariance ellipsoid on symplectic subspaces of the phase space. We compare these geometric invariants to the algebraic "universal quantum invariants" of Dodonov and Serafini.
\end{abstract}

\section{Introduction}

We consider a continuous variable system with $n$ degrees of freedom described by a Hermitian positive operator $\hat{\rho}$ on $L^{2}\left(\mathbb{R}^{n}\right)$ with trace one. The phase space of the system is identified with $\mathbb{R}^{2 n} \equiv \mathbb{R}_{x}^{n} \times \mathbb{R}_{p}^{n}$ equipped with the standard symplectic structure $z \wedge z^{\prime}=\left(z^{\prime}\right)^{T} \Omega z$ where $z=(x, p)$ and $\Omega=\left(\begin{array}{cc}0 & I \\ -I & 0\end{array}\right)$. We will prove in this paper the following geometric result: let $\mathbb{F}_{2 k}$ be an arbitrary symplectic subspace of $\mathbb{R}^{2 n}$, and denote by $\Pi_{\mathbb{F}_{2 k}}$

*maurice.de.gosson@gmail.com 
the orthogonal projection operator of $\mathbb{R}^{2 n}$ onto $\mathbb{F}_{2 k}$. Let $\sigma$ be a covariance matrix satisfying the uncertainty problem in its strong form $\sigma+\frac{1}{2} i \hbar \Omega \geq 0$ and let $W_{\sigma}: \frac{1}{2} z^{T} \sigma^{-1} z \leq 1$ be the covariance ellipsoid of the system. Let us project this ellipsoid on $\mathbb{F}_{2 k}$. The volume of this projection satisfies

$$
\operatorname{Vol}\left(\Pi_{\mathbb{F}_{2 k}} W_{\sigma}\right) \geq \frac{h^{k}}{2^{k} k !} .
$$

In particular, if $\mathbb{F}_{2 k}$ is a plane of conjugate variables $x_{j}, p_{j}$ (hence $k=1$ ) the projection is an ellipse of area not inferior to $\frac{1}{2} h$, which is a geometric form of the uncertainty principle as we have shown in previous work $([5,6,7,10])$. In addition we compare our results to Dodonov's and Serafini's "quantum universal invariants" $[3,14,15]$, which comforts us in our belief that the "Angel of Geometry" should always be preferred to the "Demon of Algebra" in conceptual questions.

Acknowledgement 1 The author has been financed by the Austrian Research Agency FWF (Projektnummer P20442-N13).

\section{Strong Form of the Uncertainty Principle}

In what follows we will write the covariance matrix as

$$
\sigma=\left(\begin{array}{cc}
\sigma_{X X} & \sigma_{X P} \\
\sigma_{X P}^{T} & \sigma_{P P}
\end{array}\right)
$$

where $\sigma_{X X}=\left(\sigma_{X_{j} X_{k}}\right)_{1 \leq j, k \leq n}, \sigma_{X P}=\sigma_{P X}^{T}=\left(\sigma_{X_{j} P_{k}}\right)_{1 \leq j, k \leq n}, \sigma_{P P}=$ $\left(\sigma_{P_{j} P_{k}}\right)_{1 \leq j, k \leq n}$ are $n \times n$ matrices $\left(\sigma_{X X}\right.$ and $\sigma_{P P}$ are symmetric).

A strong form of the uncertainty principle reads

$$
\sigma+\frac{1}{2} i \hbar \Omega \geq 0
$$

which is shorthand for saying that the Hermitian matrix $\sigma+\frac{1}{2} i \hbar \Omega$ is positive semidefinite. It is well-known that this condition implies (but is not equivalent to) the Robertson-Schrödinger inequalities

$$
\sigma_{X_{j}}^{2} \sigma_{P_{j}}^{2} \geq \sigma_{X_{j} P_{j}}^{2}+\frac{1}{4} \hbar^{2}
$$

where $\sigma_{X_{j}}^{2}=\sigma_{X_{j} X_{j}}$ and $\sigma_{P_{j}}^{2}=\sigma_{P_{j} P_{j}}$. It also implies that the covariance matrix is positive definite. The symplectic spectrum of $\sigma$ is defined as follows: the product $\Omega \sigma$ is similar to the antisymmetric matrix $\sigma^{1 / 2} \Omega \sigma^{1 / 2}$ whose eigenvalues are of the type $\pm i \nu_{j}, \nu_{j}>0$. The symplectic spectrum of 
the covariance matrix is then the sequence $\left(\nu_{1}, \ldots, \nu_{n}\right)$; the numbers $\nu_{j}$ are called the symplectic eigenvalues of $\sigma$. An important property is that the $\nu_{j}$ are symplectic invariants, i.e. they do not change under linear symplectic changes of coordinates. This is because the eigenvalues of $\Omega\left(S^{T} \sigma S\right)$ are the same as those of $\Omega \sigma$ since $\Omega\left(S^{T} \sigma S\right)=S^{-1}(\Omega \sigma) S$ in view of the relation $S \Omega S^{T}=\Omega$ characterizing linear symplectic transformations.

\section{Topological Form of the UP}

We have shown in $[7,11,8]$ that condition (2) is equivalent to

$$
c_{\mathrm{GR}}\left(W_{\sigma}\right) \geq \pi \hbar=\frac{1}{2} h
$$

where $c_{\mathrm{GR}}\left(W_{\sigma}\right)$ is the Gromov width (or: symplectic capacity $[6,11]$ ) of the covariance ellipsoid $W_{\sigma}: \frac{1}{2} z^{T} \sigma^{-1} z \leq 1$. The argument goes as follows: the Gromov width $c(W)$ of a subset $W$ of phase space $\mathbb{R}^{2 n}$ is the supremum of all numbers $\pi R^{2}$ such that the ball $B_{R}:|z| \leq R$ can be send inside $W$ using symplectic transformations, linear or not (the notion is related to Gromov's famous symplectic non-squeezing theorem [12] from 1985; see [6, 11, 10] for a review). In view of Williamson's diagonalization theorem [16] there exists $S \in \operatorname{Sp}(2 n, \mathbb{R})$ such that

$$
S^{T} \sigma S=\left(\begin{array}{cc}
\Lambda & 0 \\
0 & \Lambda
\end{array}\right)
$$

where $\Lambda$ is the diagonal matrix consisting of the symplectic eigenvalues $\nu_{j}, j=1, \ldots, n$, of the eigenvalues of the covariance matrix. Symplectic capacities are symplectic invariants, hence it is sufficient to assume that $\sigma=\left(\begin{array}{cc}\Lambda & 0 \\ 0 & \Lambda\end{array}\right)$ which reduces the proof to the case where the ellipsoid $W_{\sigma}$ is given by

$$
\sum_{j=1}^{n} \frac{1}{2 \nu_{j}}\left(x_{j}^{2}+p_{j}^{2}\right) \leq 1 .
$$

Assume now that we can squeeze the ball $B_{R}$ inside $W_{\sigma}$; this requires that the projection of that ball onto each plane $x_{j}, p_{j}$ of conjugate coordinates has radius $R \leq \sqrt{2 \nu_{j}}$, for each $j$ and hence $R \leq \sqrt{2 \nu_{\min }}$ where $\nu_{\min }=$ $\inf \left\{\nu_{1}, \ldots, \nu_{n}\right\}$; it follows that $c\left(W_{\sigma}\right)=\pi \nu_{\text {min }}$. To prove the inequality (4) it is thus sufficient to show that $\nu_{\min } \geq \frac{1}{2} \hbar$. We now exploit the condition (2). Noting that the matrix $\sigma+\frac{1}{2} i \hbar \Omega \geq 0$ is similar to $+\frac{1}{2} i \hbar \sigma^{-1 / 2} \Omega \sigma^{-1 / 2}$ this condition equivalent to

$$
I+\frac{1}{2} i \hbar \sigma^{-1 / 2} \Omega \sigma^{-1 / 2} \geq 0 .
$$


The characteristic polynomial of the matrix in the left hand side is a product

$$
P(t)=P_{1}(t) \cdots P_{n}(t)
$$

of quadratic polynomials

$$
P_{j}(t)=t^{2}-2 t+1-\frac{\hbar^{2}}{4 \nu_{j}^{2}}
$$

it follows that all the eigenvalues of that matrix are non-negative if and only if $1 \geq \hbar^{2} / 4 \nu_{j}^{2}$ for all $j=1, \ldots, n$, that is is to

$$
\nu_{j} \geq \frac{1}{2} \hbar
$$

\section{The Symplectic Camel}

One way of stating Gromov's symplectic non-squeezing theorem is the following: consider the phase space ball $B_{R}:|z| \leq R$; the orthogonal projection of $B_{R}$ on any of the conjugate coordinates planes $x_{j}, p_{j}$ is the circle $x_{j}^{2}+p_{j}^{2} \leq R^{2}$, which has area $\pi R^{2}$. Suppose we deform $B_{R}$ using a canonical transformation $f$; the set $f\left(B_{R}\right)$ will have same volume as $B_{R}$ (Liouville's theorem), but, in addition, the orthogonal projection of $f\left(B_{R}\right)$ on the planes $x_{j}, p_{j}$ will always have an area at least equal to $\pi R^{2}$ (but the area of the projection on planes of non-conjugate variables can take arbitrarily small values). An extension of the non-squeezing theorem has recently be proved by Abbondandolo and Matveyev [1]. It is well-understood only in the linear (and affine) case, but this is sufficient for our purposes. Let $\mathbb{R}^{2 k}$ $(1 \leq k \leq n)$ be a symplectic subspace of $\mathbb{R}^{2 n}$. This means that the restriction of the symplectic product $\wedge$ to $\mathbb{R}^{2 k}$ is non-degenerate, and hence itself a symplectic product. An elementary example is the set of all coordinates $\left\{\left(x_{1}, \ldots, x_{k} ; p_{1}, \ldots, p_{k}\right)\right\}$; in fact every symplectic subspace can be obtained from the latter using linear symplectic transformations. Let us denote by $B^{2 k}(R)$ the ball with radius $R$ centered at the origin in $\mathbb{R}^{2 k}$; its volume is $\left(\pi R^{2}\right)^{k} / k$ ! . Let now $\Pi_{k}$ be the orthogonal projection of $\mathbb{R}^{2 n}$ onto $\mathbb{R}^{2 k}$; obviously $\Pi_{k} B^{2 n}(R)=B^{2 k}(R)$. What Abbondandolo and Matveyev prove is that for very $S$ in $\operatorname{Sp}(2 n, \mathbb{R})$ following inequality holds:

$$
\operatorname{Vol} \Pi_{k}\left(S B^{2 n}(R)\right) \geq \operatorname{Vol} \Pi_{k}\left(B^{2 n}(R)\right)=\frac{\left(\pi R^{2}\right)^{k}}{k !} .
$$

When $k=n$ this inequality is trivial (it actually becomes an equality because symplectic transformations are volume-preserving), and for $k=1$ it 
is a reformulation of Gromov's non-squeezing theorem in the linear case. The interesting point with this formula is that it provides us with a statement valid for middle-dimensional symplectic spaces. Abbondandolo and Matveyev give an example in [1] showing that the inequality (9) is not always true for $k$ between 1 and $n$ if we replace $S$ by an arbitrary non-linear symplectic transformation; their counterexample is however of a rather pathological nature, so one may conjecture that (9) holds for quite large classes of nonlinear symplectic transformations. This conjecture has not yet been proved, and is an active area of research.

\section{Geometric Invariants: the Main Result}

The inequality (9) allows us to prove the main result of this paper: assume that we project orthogonally the covariance ellipsoid $W_{\sigma}: \frac{1}{2} z^{T} \sigma^{-1} z \leq 1$ on a $2 k$-dimensional symplectic subspace $\mathbb{F}_{2 k}$ of $\mathbb{R}^{2 n}$; the projection is an ellipsoid $\Pi_{\mathbb{F}_{2 k}} W_{\sigma}$ in $\mathbb{F}_{2 k}$ whose volume

$$
\operatorname{Vol}\left(\Pi_{\mathbb{F}_{2 k}} W_{\sigma}\right)=\frac{(2 \pi)^{k}}{k !} \nu_{1} \cdots \nu_{k}
$$

satisfies the inequality

$$
\operatorname{Vol}\left(\Pi_{\mathbb{F}_{2 k}} W_{\sigma}\right) \geq \frac{h^{k}}{2^{k} k !}
$$

We first remark that it suffices to prove the inequality (11) for one $2 k$-dimensional symplectic subspace $\mathbb{F}_{2 k}$ for it will then hold for all such subspaces. Let in fact $\mathbb{F}_{2 k}^{\prime}$ be another such subspace with same dimension, and choose symplectic bases $\mathcal{B}_{2 k}=\left\{e_{1}, \ldots, e_{k} ; f_{1}, \ldots, f_{k}\right\}$ and $\mathcal{B}_{2 k}^{\prime}=$ $\left\{e_{1}^{\prime}, \ldots, e_{k}^{\prime} ; f_{1}^{\prime}, \ldots, f_{k}^{\prime}\right\}$ of $\mathbb{F}_{2 k}$ and $\mathbb{F}_{2 k}^{\prime}$, respectively (i.e. $e_{i} \wedge e_{j}=f_{i} \wedge f_{j}=0$, $f_{i} \wedge e_{j}=\delta_{i j}$ for $1 \leq i, j \leq k$, and similar relations for the elements of $\mathcal{B}_{k}^{\prime}$ ). Completing $\mathcal{B}_{2 k}$ and $\mathcal{B}_{2 k}^{\prime}$ to full symplectic bases $\mathcal{B}_{2 n}$ and $\mathcal{B}_{2 n}^{\prime}$ of $\mathbb{R}^{2 n}$ (the "symplectic Gram-Schmidt theorem", see e.g. [6], Ch. 1) the linear automorphism $S$ of $\mathbb{R}^{2 n}$ defined by $S\left(e_{j}\right)=e_{j}^{\prime}, S\left(f_{j}\right)=f_{j}^{\prime}$ for $1 \leq j \leq n$ is symplectic, and so is its restriction $S_{k}$ to the subspace $\mathbb{F}_{2 k}$. Symplectic mappings being volume preserving, $S_{k}$ sends $\Pi_{\mathbb{F}_{2 k}} W_{\sigma}$ to an ellipsoid with same volume as $\Pi_{\mathbb{F}_{2 k}} W_{\sigma}$. Suppose now the covariance matrix diagonal of

the type $\lambda=\left(\begin{array}{cc}\Lambda & 0 \\ 0 & \Lambda\end{array}\right)$; then, choosing $\mathbb{F}_{2 k}=\mathbb{R}^{2 k}$, identified with the set of points $\left(x_{1}, \ldots, x_{k} ; p_{1}, \ldots, p_{k}\right)$, we have, using formula (5),

$$
\Pi_{\mathbb{R}^{2 k}} W_{\lambda}: \sum_{j=1}^{k} \frac{1}{2 \nu_{j}}\left(x_{j}^{2}+p_{j}^{2}\right) \leq 1 .
$$


The volume of this projected ellipsoid is

$$
\operatorname{Vol}\left(\Pi_{\mathbb{R}^{2 k}} W_{\lambda}\right)=\frac{(2 \pi)^{k}}{k !} \nu_{1} \cdots \nu_{k}
$$

and the inequality (11) follows in this case since $\nu_{j} \geq \frac{1}{2} \hbar$ for all indices $j$. The general case (11) is easily deduced. We first note that in view of the discussion of symplectic spaces above, the inequality (12) is preserved if we replace $\mathbb{R}^{2 k}$ with an arbitrary symplectic subspace $\mathbb{F}_{2 k}$. Next, writing $\sigma=S^{T} \lambda S$ (Williamson's diagonal form [16]) we have $W_{\sigma}=S^{T} W_{\lambda}$; the claim now follows from the Abbondandolo and Matveyev inequality (9).

\section{Discussion}

In $[14,15]$ Serafini studies the "universal symplectic invariants" introduced by Dodonov [3]. Working in units in which $\hbar=2$, he denotes by $\Delta_{j}^{n}$ the principal minor of order $2 j$ of $\Omega \sigma$, i.e., the sum of the determinants of all the principal submatrices of order $2 j$ of $\Omega \sigma$ (by convention $\Delta_{0}^{n}=1$ ). It is easy to check that the numbers $\Delta_{j}^{n}$ are just the coefficients of the characteristic polynomial of the matrix $\Omega \sigma$, and hence

$$
\Delta_{j}^{n}=\sum_{C(n, j)}\left(\prod_{k \in C(n, j)} \nu_{k}^{2}\right)
$$

where the sum runs over all the possible combinations $C(n, j)$ of $j$ integers $\leq n$. A rapid comparison of formulas (12) and (13) shows that the numbers $\Delta_{k}^{n}$ are related to the volumes of the projections of the covariance ellipsoid by the formula

$$
\Delta_{j}^{n}=\left(\frac{j^{!}}{(2 \pi)^{j}}\right)^{2} \sum_{\mathbb{F}_{2 j} \in \mathrm{Sp}_{2 j}}\left(\Pi_{\mathbb{F}_{2 j}} W_{\sigma}\right)^{2}
$$

where $\mathrm{Sp}_{2 k}$ is the set of all $n ! / j !(n-j)$ ! symplectic coordinate subspaces of $\mathbb{R}^{2 n}$.

The terms $\left(\Pi_{\mathbb{F}_{2 j}} W_{\sigma}\right)^{2}$ which are of a geometric nature contain more information that the $\Delta_{j}^{n}$ which are defined in algebraic terms, and should thus given a privileged status when studying invariants of continuous variables quantum systems. 


\section{References}

[1] A. Abbondandolo and S. Matveyev, How large is the shadow of a symplectic ball? J Topol. Anal. 05(01), (2013); Preprint arXiv:1202.3614v1 [math.SG]

[2] V. I. Arnold, Mathematical Methods of Classical Mechanics, Graduate Texts in Mathematics, 2nd edition, Springer-Verlag, (1989)

[3] V. V. Dodonov, Universal integrals of motion and universal invariants of quantum systems. J. Phys. A: Mathematical and General 33(43), 7721 (2000)

[4] V. V. Dodonov, E. V. Kurmyshev, and V. I. Man'ko, Generalized uncertainty relation and correlated coherent states. Phys. Lett. A, 79(2-3), 150-152 (1980)

[5] M. de Gosson, Phase Space Quantization and the Uncertainty Principle. Phys. Lett. A, 317/5-6, 365-369 (2003)

[6] M. de Gosson, Symplectic Geometry and Quantum Mechanics, series "Operator Theory: Advances and Applications" Vol. 166, Birkhäuser, Basel (2006)

[7] M. de Gosson, The Symplectic Camel and the Uncertainty Principle: The Tip of an Iceberg? Found. Phys. 99, 194-214 (2009)

[8] M. de Gosson, On the use of minimum volume ellipsoids and symplectic capacities for studying classical uncertainties for joint positionmomentum measurements. J. Stat. Mech. P11005 (2010)

[9] M. de Gosson, Quantum Blobs. Found. Phys. 43:440-457 (2012)

[10] M. de Gosson, The Symplectic Egg in Classical and Quantum Mechanics, to appear in Amer. J. Phys. (2013), prelim. version: arXiv:1208.5969v1 [math-ph]

[11] M. de Gosson and F. Luef, Symplectic capacities and the geometry of uncertainty: The irruption of symplectic topology in classical and quantum mechanics. Phys. Reps. 484(5), 131-179 (2009)

[12] M. Gromov, Pseudoholomorphic curves in symplectic manifolds. Inventiones Mathematica 82, 307-347 (1985) 
[13] L. Polterovich, The Geometry of the Group of Symplectic Diffeomorphisms. Lectures in Mathematics, Birkhäuser (2001)

[14] A. Serafini, Multimode Uncertainty Relations and Separability of Continuous Variable States. Phys. Rev. Lett. 96, 110402-1 (2006)

[15] A. Serafini, Detecting entanglement by symplectic uncertainty relations. J. Opt. Soc. Am. B, 24(3), 347-354 (2007)

[16] J. Williamson, On the algebraic problem concerning the normal forms of linear dynamical systems, Amer. J. of Math. 58, 141-163 (1936) 